Supporting Information for:

\title{
Stepwise Coordination Assembly Approach toward Aluminum-lanthanide-based Compounds
}

San-Tai Wang, ${ }^{\dagger \neq}$ Shu-Hua Zhang, ${ }^{\ddagger}$ Wei-Hui Fang, ${ }^{* \dagger}$ and Jian Zhang*+

${ }^{+}$State Key Laboratory of Structural Chemistry, Fujian Institute of Research on the Structure of Matter, Chinese Academy of Sciences, Fuzhou, Fujian 350002, China.

${ }^{\ddagger}$ College of Chemistry, Guilin University of Technology, Guilin, Guangxi 541004, China.

Corresponding Author

*E-mail: fwh@fjirsm.ac.cn; zhj@fjirsm.ac.cn 


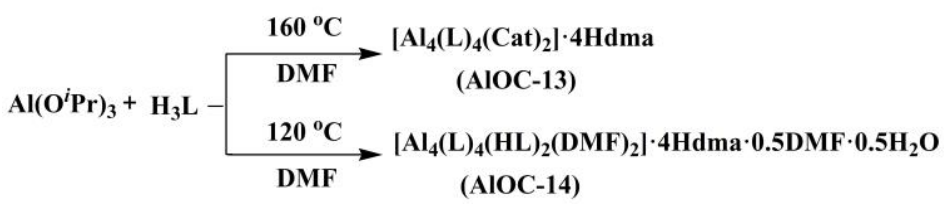

Figure S1. Synthetic diagram of AlOC-13 and AlOC-14.

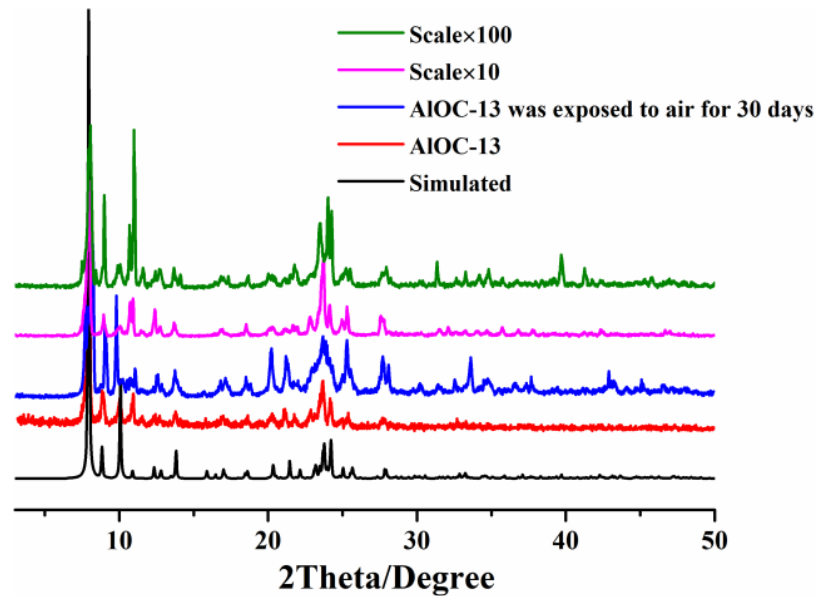

Figure S2. The PXRD of the simulated, experimental patterns, keep in the air 30 days and scale-up synthesis of AlOC-13.

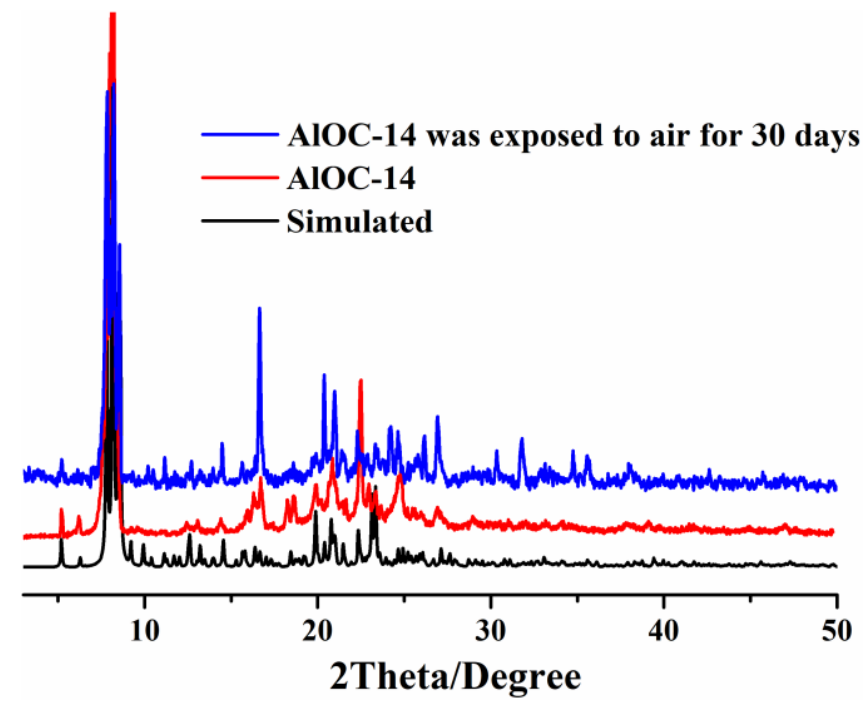

Figure S3. The PXRD of the simulated, experimental patterns and keep in the air 30 days of AlOC-14. 
(a)

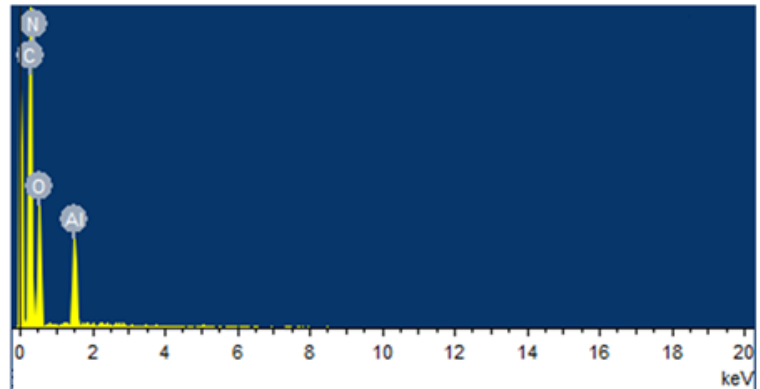

(b)

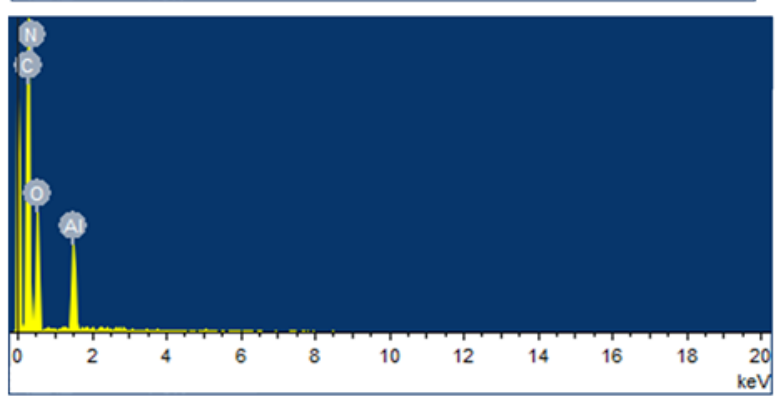

Figure S4. The EDS spectrum of AlOC-13 (a) and AlOC-14 (b).

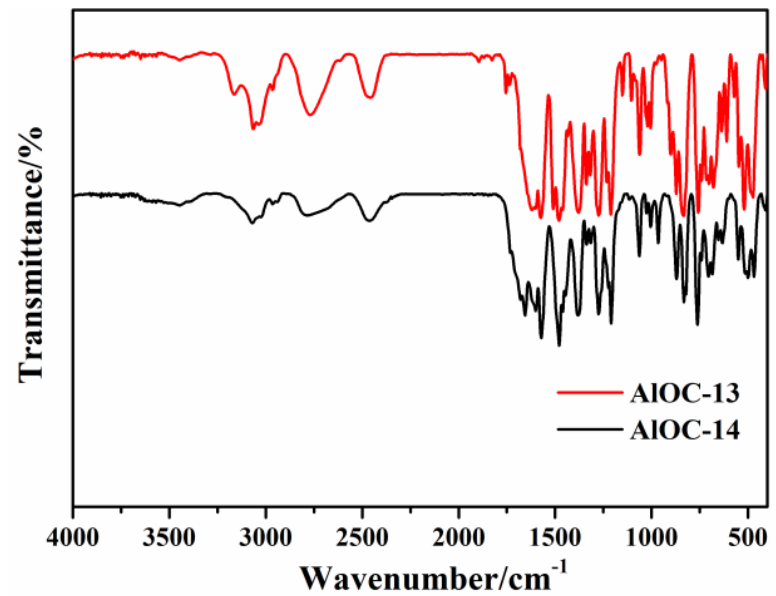

Figure S5. The IR spectrum of AlOC-13 and AlOC-14.

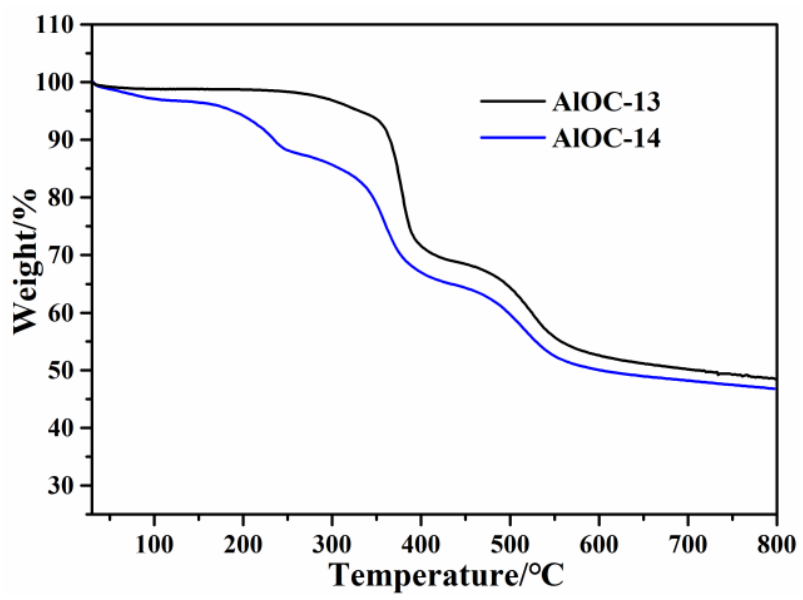

Figure S6. The TGA curve of AlOC-13 and AlOC-14. 


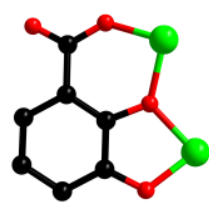

$\mathrm{L}^{3-}$

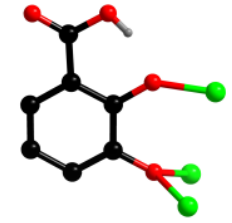

$\mathrm{HL}^{2-}$

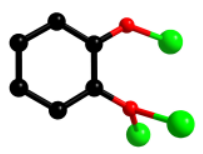

$\mathrm{Cat}^{2-}$

Figure S7. Coordination mode I $\left(\eta_{1}: \eta^{2}: \eta^{1}: \mu^{2}\right)$, II $\left(\eta^{1}: \eta^{2}: \mu^{3}\right)$ of $L^{3-}, \mathrm{HL}^{2-}$ and Cat ${ }^{2-}$.

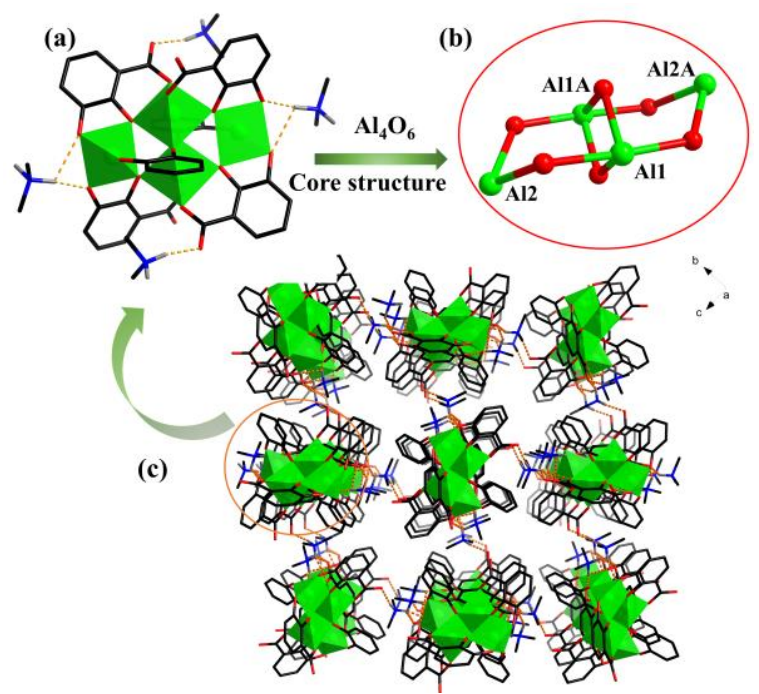

Figure S8. (a) Crystal structure of AlOC-13 based on $\mathrm{H}_{3} \mathrm{~L}$ ligand (b) the core structure in AlOC-13, symmetry codes: (i) 1-x, 1-y, 1-z. (c) the packing diagram for AlOC-13 viewed along a-direction (Atom color code: green, Al; red, O; black, C; blue, $\mathrm{N}$; grey, $\mathrm{H})$.
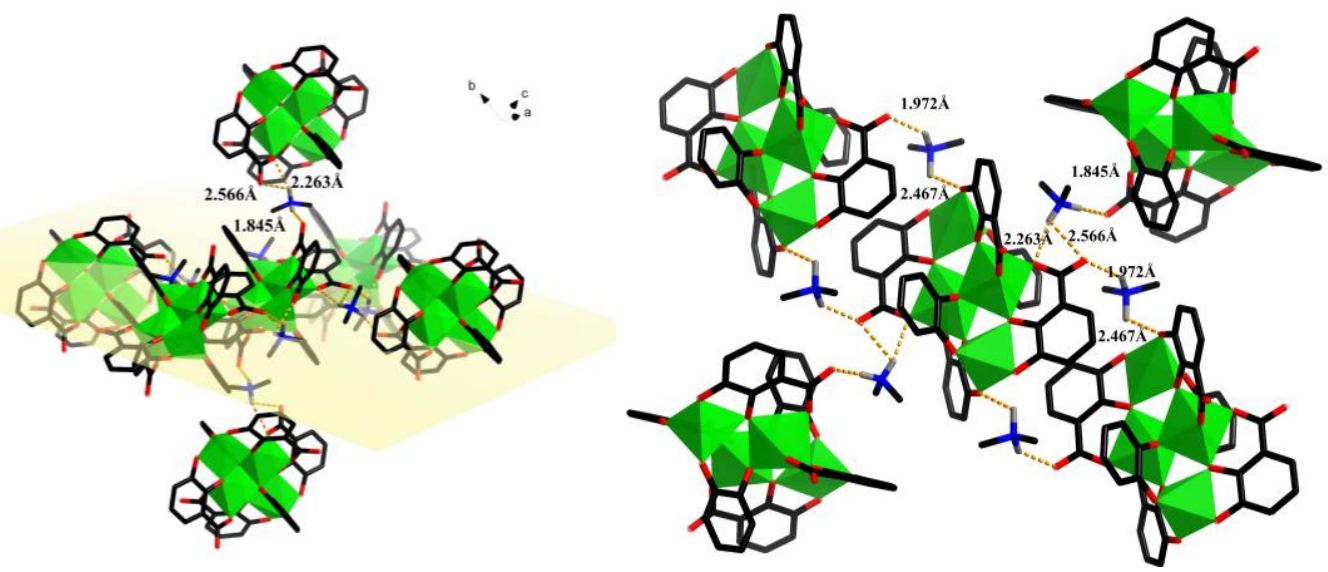

Figure S9. The hydrogen bonding in AlOC-13. 


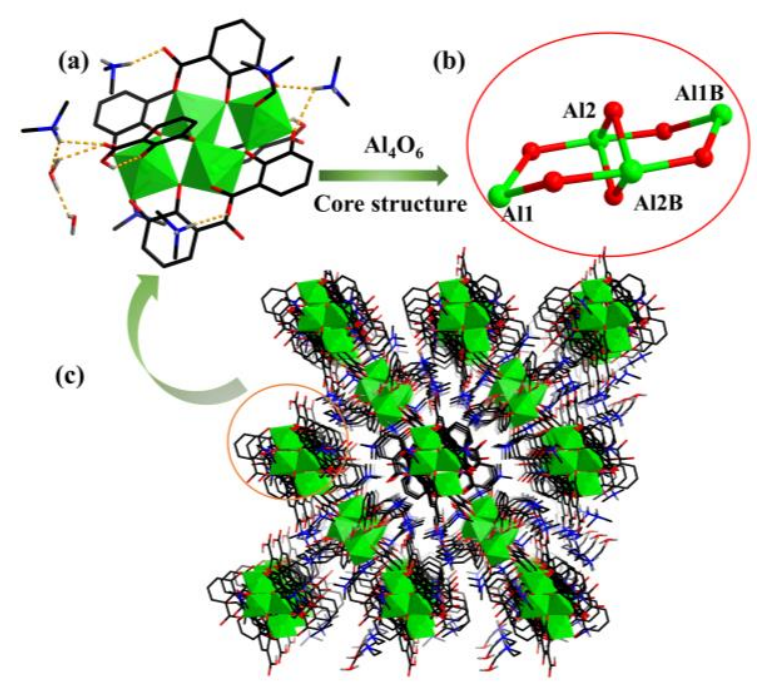

Figure S10. (a) Crystal structure of AlOC-14 based on $\mathrm{H}_{3} \mathrm{~L}$ ligand (b) the core structure in AlOC-14, (Symmetry codes: (i) $-x+1,-y+1,-z+1$; (ii) $-x,-y+2,-z)$. (c) the packing diagram for AlOC-14 viewed along c-direction (Atom color code: green, $\mathrm{Al}$; red, O; black, C; blue, $\mathrm{N}$; grey, $\mathrm{H}$ ).

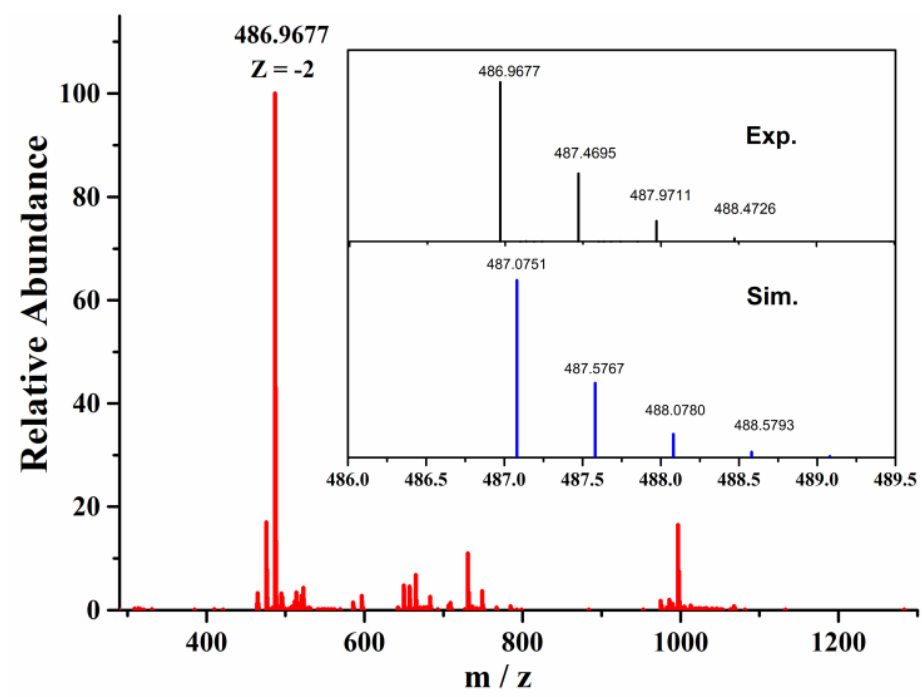

Figure S1 1. Negative-mode ESI-MS spectra of the $\mathrm{H}_{2} \mathrm{O}$ solutions of AlOC-13, highlighting the comparison of experimental isotopic envelops (black) with simulated patterns (blue) of $\left[\mathrm{Al}_{4}(\mathrm{~L})_{3}(\mathrm{OH})_{3}(\mathrm{H} 2 \mathrm{O})_{3}(\mathrm{Cat})_{2}(\mathrm{Hdma})_{2}\right]^{2-}$ for AlOC-13.

ESI-MS spectrum of the $\mathrm{H}_{2} \mathrm{O}$ solution of AlOC-13 shows a dominant signal of $\mathrm{m} / \mathrm{z} 486.9677$ which can be assigned to a species of $\left[\mathrm{Al}_{4}(\mathrm{~L})_{3}(\mathrm{OH})_{3}\left(\mathrm{H}_{2} \mathrm{O}\right)_{3}(\mathrm{Cat})_{2}(\mathrm{Hdma})_{2}\right]^{2-}(\mathrm{m} / \mathrm{z}$ 487.0750) (Figure 2a). Moreover, the results of ESI-MS showed that although one $\mathrm{L}^{3-}$ ligand of AlOC-13 was shed during the dissolution process in water, the whole remained stable. the characteristic isotopic envelopes of the experimental and simulated patterns match very well, further confirming the solution stability of AlOC-13 in $\mathrm{H}_{2} \mathrm{O}$. 
(a)

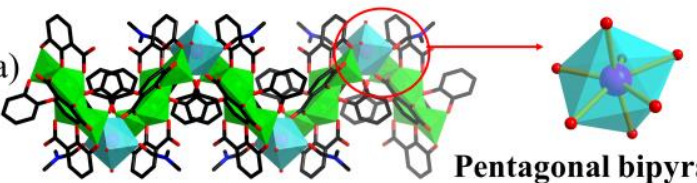

(b)

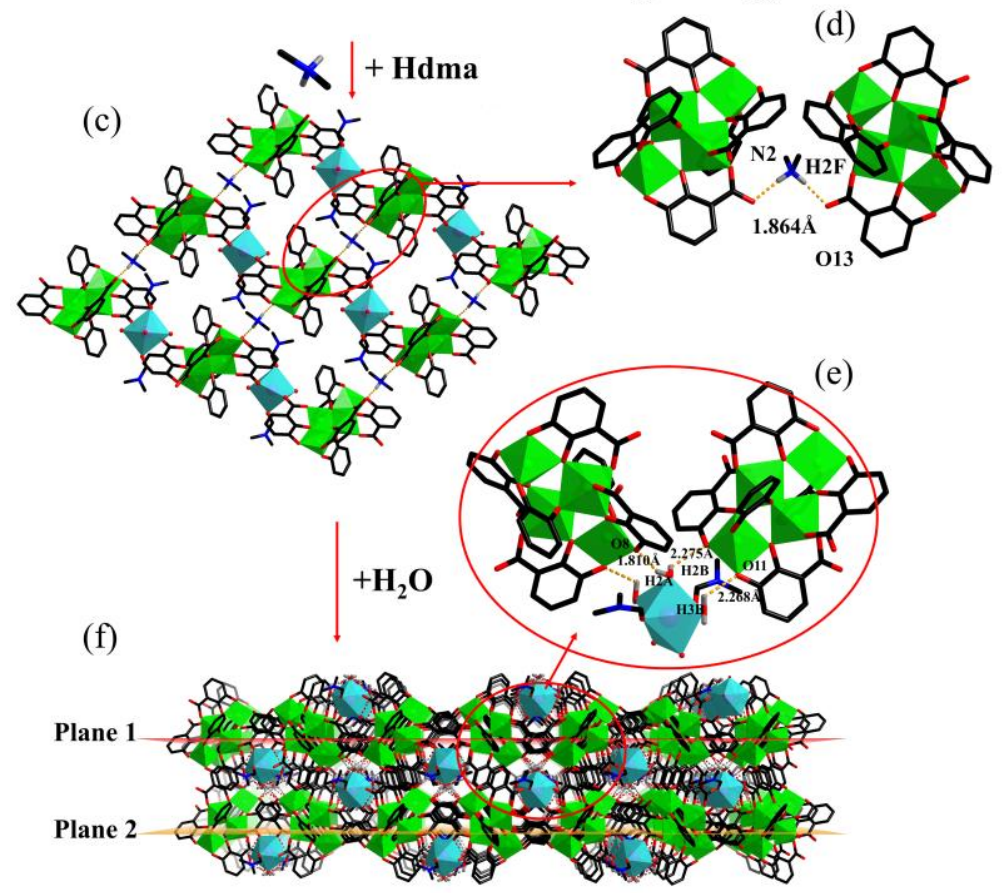

Figure S12. (a) 1D "Z" chain of AlOC-13 through $\mathrm{Ln}^{3+}$ coordination. (b) coordination environment of $\mathrm{Ln}^{3+}$ ions (c) the 2D hydrogen bonding plane. (d) Hydrogen bonding between protonated dimethylamine and adjacent one-dimensional chain (e) O-H $\cdots \mathrm{O}$ hydrogen bonding between plane 1 and plane 2 (f) 3D hydrogen bonding framework for AlOC-13-Ln viewed along a-direction. (orange dotted lines represent hydrogen bonds)

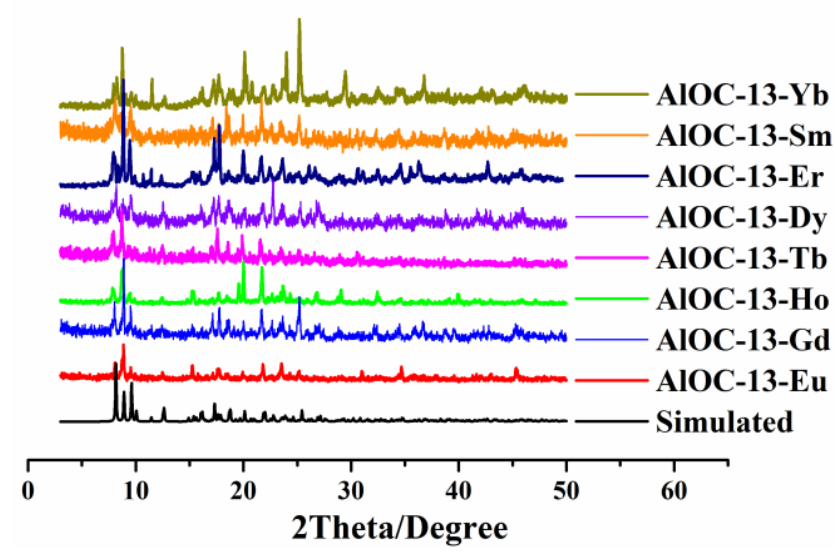

Figure S13. The PXRD of the simulated and experimental patterns of AlOC-13- $\mathrm{Ln}(\mathrm{Ln}=\mathrm{Sm}, \mathrm{Eu}, \mathrm{Gd}, \mathrm{Tb}, \mathrm{Dy}, \mathrm{Ho}, \mathrm{Er}, \mathrm{Yb})$. 


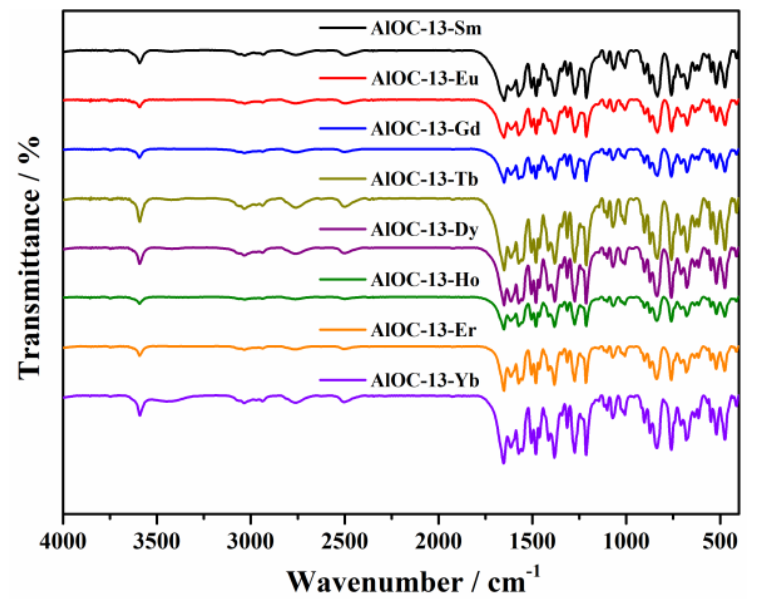

Figure S14. The IR spectrum of AlOC-13-Ln ( $\mathrm{Ln}=\mathrm{Sm}, \mathrm{Eu}, \mathrm{Gd}, \mathrm{Tb}, \mathrm{Dy}, \mathrm{Ho}, \mathrm{Er}, \mathrm{Yb})$.

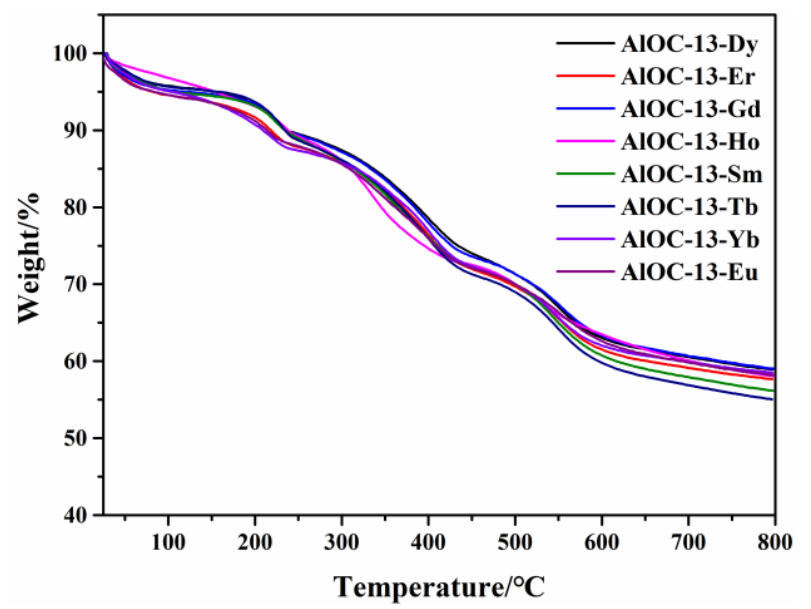

Figure S15. The TGA curve for AlOC-13-Ln series ( $\mathrm{Ln}=\mathrm{Sm}, \mathrm{Eu}, \mathrm{Gd}, \mathrm{Tb}, \mathrm{Dy}, \mathrm{Ho}, \mathrm{Er}, \mathrm{Yb})$.

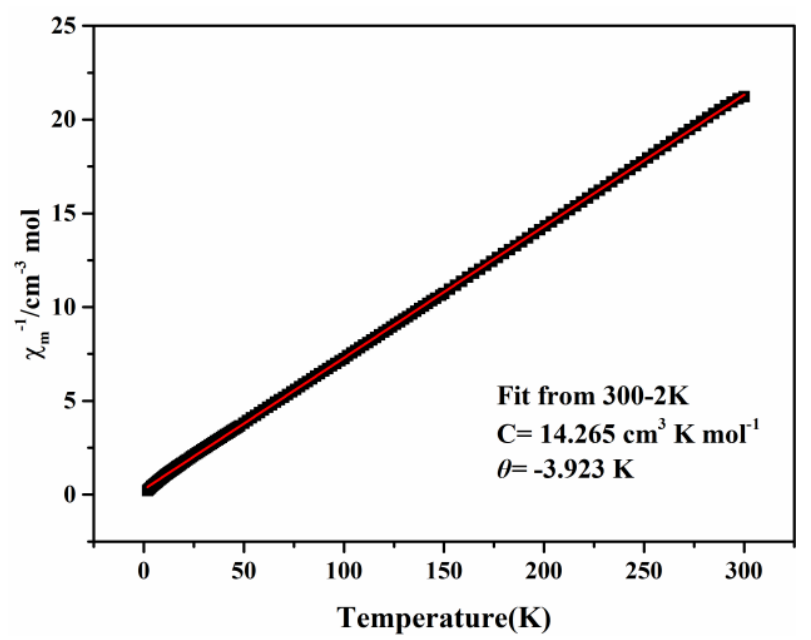

Figure S16. The temperature dependence of the reciprocal susceptibility $\left(1 / \chi_{\mathrm{m}}\right)$ for AlOC-13-Dy. 


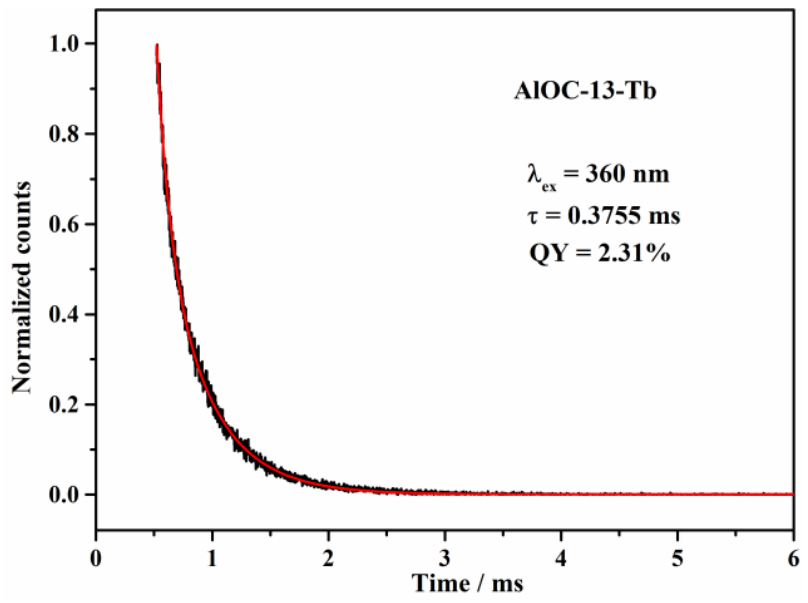

Figure S17. The fluorescence lifetime curve of AlOC-13-Tb. 
Table S1. Selected bond lengths $(\AA)$ and angles $\left(^{\circ}\right)$ for AlOC-13.

\begin{tabular}{|c|c|c|c|c|c|}
\hline Al1-O5 & $1.8717(16)$ & $\mathrm{O}^{\mathrm{i}}-\mathrm{A} 11-\mathrm{O} 8^{\mathrm{i}}$ & $84.78(7)$ & $\mathrm{O} 4-\mathrm{A} 12-\mathrm{O} 3$ & $83.34(7)$ \\
\hline $\mathrm{All}-\mathrm{OS}^{\mathrm{i}}$ & $1.8762(16)$ & O5-Al1-O8 & $90.36(7)$ & $\mathrm{O} 4-\mathrm{Al} 2-\mathrm{O} 7$ & $87.91(8)$ \\
\hline Al1-O ${ }^{\mathrm{i}}$ & $1.9182(16)$ & O5-Al1-O3 & $84.66(7)$ & O7-Al2-O8 & $83.60(7)$ \\
\hline Al1-O3 & $1.9059(16)$ & $\mathrm{O}^{\mathrm{i}}-\mathrm{Al} 1-\mathrm{O} 3$ & $89.84(7)$ & $\mathrm{O} 7-\mathrm{A} 12-\mathrm{O} 3$ & $155.12(8)$ \\
\hline $\mathrm{Al1}-\mathrm{O} 2$ & $1.8372(17)$ & O3-Al1-O ${ }^{\mathrm{i}}$ & $173.35(8)$ & O2-Al1-O5 & $93.86(7)$ \\
\hline Al1-O10 & $1.8468(17)$ & O2-Al1-O5 & $170.86(8)$ & O2-Al1-O ${ }^{\mathrm{i}}$ & $92.72(7)$ \\
\hline $\mathrm{Al} 2-\mathrm{O} 8$ & $1.9184(17)$ & $\mathrm{O} 8-\mathrm{A} 12-\mathrm{O} 3$ & $88.84(7)$ & $\mathrm{O} 2-\mathrm{A} 11-\mathrm{O} 3$ & $91.53(7)$ \\
\hline $\mathrm{Al} 2-\mathrm{O} 3$ & $1.9444(17)$ & O6-A12-O8 & $112.73(8)$ & $\mathrm{O} 2-\mathrm{Al1}-\mathrm{O} 10^{\mathrm{i}}$ & $94.58(8)$ \\
\hline $\mathrm{Al} 2-\mathrm{O} 6$ & $1.7599(18)$ & O6-A12-O3 & $103.27(8)$ & O10-Al1-O5 & $94.29(7)$ \\
\hline $\mathrm{Al} 2-\mathrm{O} 4$ & $1.8250(17)$ & O6-A12-O4 & $105.75(8)$ & O10 $-\mathrm{Al1}-\mathrm{OS}^{\mathrm{i}}$ & $171.09(8)$ \\
\hline $\mathrm{Al} 2-\mathrm{O} 7$ & $1.8477(18)$ & O6-A12-O7 & $101.52(9)$ & O10 $-A 11-O 8^{i}$ & $92.16(7)$ \\
\hline O5-Al1-OS & $77.40(7)$ & O4-A12-O8 & $141.50(8)$ & O10 - Al1-O3 & $92.59(7)$ \\
\hline
\end{tabular}

Symmetry codes: (i) $1-x, 1-y, 1-z$ (ii) $x-1 / 2,-y+2 / 3, z-1 / 2$ (iii) $-x+2,-y+1,-z+1$.

Table S2. Selected bond lengths $(\AA)$ and angles $\left(^{\circ}\right)$ for AlOC-14.

\begin{tabular}{|c|c|c|c|c|c|}
\hline $\mathrm{Al} 4-\mathrm{O}^{\mathrm{i}}$ & $1.8744(19)$ & O7-A14-O3 & $176.87(8)$ & $\mathrm{O} 17-\mathrm{A} 12-\mathrm{O} 26$ & $175.47(10)$ \\
\hline $\mathrm{Al} 4-\mathrm{O} 5$ & $1.8862(18)$ & O2-A14-O5 & $93.85(9)$ & $\mathrm{O} 25-\mathrm{A} 12-\mathrm{O} 24$ & $94.88(9)$ \\
\hline $\mathrm{Al} 4-\mathrm{O} 7$ & $1.9065(18)$ & $\mathrm{O} 2-\mathrm{Al} 4-\mathrm{O}^{\mathrm{i}}$ & $170.18(9)$ & $\mathrm{O} 25-\mathrm{A} 12-\mathrm{O} 24^{\mathrm{ii}}$ & $170.41(10)$ \\
\hline $\mathrm{Al} 4-\mathrm{O} 2$ & $1.847(2)$ & O2-A14-O7 & $91.46(8)$ & $\mathrm{O} 25-\mathrm{A} 12-\mathrm{O} 17$ & $92.75(9)$ \\
\hline $\mathrm{Al} 4-\mathrm{O} 3$ & $1.9074(17)$ & $\mathrm{O} 2-\mathrm{A} 14-\mathrm{O} 3$ & $90.95(8)$ & $\mathrm{O} 25-\mathrm{A} 12-\mathrm{O} 26$ & $91.07(9)$ \\
\hline $\mathrm{Al} 4-\mathrm{O} 4$ & $1.8464(19)$ & O2-A14-O4 & $93.77(9)$ & $\mathrm{O} 25-\mathrm{A} 12-\mathrm{O} 16$ & $93.90(10)$ \\
\hline $\mathrm{Al} 3-\mathrm{O} 7$ & $1.9216(18)$ & O4-Al4-O5 ${ }^{\mathrm{i}}$ & $95.69(9)$ & $\mathrm{O} 16-\mathrm{Al} 2-\mathrm{O} 24^{\mathrm{ii}}$ & $95.07(9)$ \\
\hline $\mathrm{Al} 3-\mathrm{O}^{\mathrm{i}}$ & $1.9211(19)$ & O4-A14-O5 & $172.05(9)$ & $\mathrm{O} 16-\mathrm{A} 12-\mathrm{O} 24$ & $170.86(10)$ \\
\hline $\mathrm{Al} 3-\mathrm{O} 8$ & $1.855(2)$ & O4-A14-O7 & $91.02(8)$ & O16-Al2-O17 & $91.02(9)$ \\
\hline $\mathrm{Al3}-\mathrm{O} 13$ & $1.834(2)$ & O4-A14-O3 & $90.81(8)$ & $\mathrm{O} 16-\mathrm{A} 12-\mathrm{O} 26$ & $91.15(9)$ \\
\hline $\mathrm{Al} 3-\mathrm{O} 12$ & $2.057(2)$ & O7-Al3-O12 & $85.08(8)$ & O17-A11-O21 & $83.08(10)$ \\
\hline $\mathrm{Al3}-\mathrm{O} 11^{\mathrm{i}}$ & $1.8569(19)$ & $\mathrm{O} 3^{\mathrm{i}}-\mathrm{Al} 3-\mathrm{O} 7$ & $95.48(8)$ & $\mathrm{O} 26^{\mathrm{ii}}-\mathrm{Al} 1-\mathrm{O} 17$ & $94.51(9)$ \\
\hline $\mathrm{Al} 2-\mathrm{O} 24^{\mathrm{ii}}$ & $1.882(2)$ & $\mathrm{O} 33^{\mathrm{i}} \mathrm{Al}-\mathrm{O} 12$ & $82.61(8)$ & $\mathrm{O} 26^{\mathrm{ii}}-\mathrm{Al} 1-\mathrm{O} 21$ & $82.83(11)$ \\
\hline $\mathrm{A} 12-\mathrm{O} 24$ & $1.883(2)$ & O8-Al3-O7 & $96.52(9)$ & O20-Al1-O17 & $96.63(10)$ \\
\hline $\mathrm{A} 12-\mathrm{O} 17$ & $1.9067(19)$ & $\mathrm{O} 8-\mathrm{Al} 3-\mathrm{O}^{\mathrm{i}}$ & $96.31(9)$ & $\mathrm{O} 20-\mathrm{A} 11-\mathrm{O} 26^{\mathrm{ii}}$ & $100.07(10)$ \\
\hline $\mathrm{A} 12-\mathrm{O} 26$ & $1.9092(19)$ & O8-Al3-O12 & $178.16(9)$ & O20-A11-O21 & $177.10(13)$ \\
\hline $\mathrm{A} 12-\mathrm{O} 25$ & $1.841(2)$ & O8-A13-O11 ${ }^{\mathrm{i}}$ & $91.57(9)$ & O20-Al1-O18A & $87.1(2)$ \\
\hline $\mathrm{Al2}-\mathrm{O} 16$ & $1.847(2)$ & O13-Al3-O7 & $86.00(8)$ & O20-Al1-O19ii & $93.33(10)$ \\
\hline Al1-O17 & $1.923(2)$ & $\mathrm{O} 13-\mathrm{Al} 3-\mathrm{O}^{\mathrm{i}}$ & $169.55(10)$ & O18B-Al1-O17 & $87.5(4)$ \\
\hline $\mathrm{Al} 1-\mathrm{O} 26^{\mathrm{ii}}$ & $1.909(2)$ & O13-Al3-O8 & $93.80(10)$ & O18B-Al1-O26 $6^{\mathrm{ii}}$ & $155.9(3)$ \\
\hline $\mathrm{Al1}-\mathrm{O} 20$ & $1.840(2)$ & $\mathrm{O} 13-\mathrm{A} 13-\mathrm{O} 12$ & $87.21(9)$ & O18B-Al1-O20 & $103.5(3)$ \\
\hline Al1-O21 & $2.099(3)$ & O13-Al3-O11 $1^{\mathrm{i}}$ & $91.75(8)$ & O18B-Al1-O21 & $73.6(3)$ \\
\hline Al1-O18B & $1.743(12)$ & $\mathrm{O} 11^{\mathrm{i}-\mathrm{Al} 3-\mathrm{O} 7}$ & $171.73(9)$ & O18B-Al1-O19i & $88.5(3)$ \\
\hline Al1-O18A & $1.905(11)$ & $\mathrm{O} 11^{\mathrm{i}}-\mathrm{A} 13-\mathrm{O} 3^{\mathrm{i}}$ & $85.33(8)$ & O18A-Al1-O17 & $84.3(3)$ \\
\hline Al1-O19 $9^{\mathrm{ii}}$ & $1.855(2)$ & $\mathrm{O} 11^{\mathrm{i}-\mathrm{A} 13-\mathrm{O} 12}$ & $86.87(9)$ & O18A-Al1-O21 & $90.0(3)$ \\
\hline $\mathrm{O}^{\mathrm{i}}-\mathrm{A} 14-\mathrm{O} 5$ & $76.81(9)$ & $\mathrm{O} 24^{\mathrm{ii}}-\mathrm{A} 2-\mathrm{O} 24$ & $76.32(9)$ & O19 ${ }^{\text {ii }-A l 1-O 17 ~}$ & $169.91(11)$ \\
\hline O5-A14-O7 & $86.53(8)$ & $\mathrm{O} 24^{\mathrm{ii}-\mathrm{A} 12-\mathrm{O} 17}$ & $90.46(9)$ & $\mathrm{O} 19^{\mathrm{ii}}-\mathrm{Al} 1-\mathrm{O} 26^{\mathrm{ii}}$ & $85.35(10)$ \\
\hline
\end{tabular}




\begin{tabular}{|c|c|c|c|c|c|}
\hline $\mathrm{O}^{\mathrm{i}}-\mathrm{Al} 4-\mathrm{O} 7$ & $90.98(8)$ & $\mathrm{O} 24-\mathrm{A} 12-\mathrm{O} 17$ & $86.04(8)$ & $\mathrm{O} 19^{\mathrm{ii}}-\mathrm{A} 11-\mathrm{O} 21$ & $86.89(11)$ \\
\hline O5-A14-O3 & $91.32(8)$ & $\mathrm{O} 24-\mathrm{A} 12-\mathrm{O} 26$ & $91.21(9)$ & O19 $9^{\mathrm{ii}}-\mathrm{A} 11-\mathrm{O} 18 \mathrm{~A}$ & $94.6(3)$ \\
\hline $\mathrm{O}^{\mathrm{i}}-\mathrm{Al} 4-\mathrm{O} 3$ & $86.31(8)$ & $\mathrm{O} 24^{\mathrm{ii}}-\mathrm{A} 12-\mathrm{O} 26$ & $85.38(9)$ & & \\
\hline
\end{tabular}

Table S3. Hydrogen bond parameters for AlOC-13.

\begin{tabular}{lllll}
\hline $\mathrm{D}-\mathrm{H} \cdots \mathrm{A}$ & $\mathrm{d}(\mathrm{D}-\mathrm{H})$ & $\mathrm{d}(\mathrm{D} \cdots \mathrm{A})$ & $\mathrm{d}(\mathrm{H} \cdots \mathrm{A})$ & $<(\mathrm{DHA})$ \\
\hline $\mathrm{N}(1)-\mathrm{H}(1 \mathrm{~A}) \cdots \mathrm{O}(1)$ & $0.95(5)$ & $2.768(4)$ & $1.85(5)$ & $162(4)$ \\
$\mathrm{N}(1)-\mathrm{H}(1 \mathrm{~B}) \cdots \mathrm{O}(9)^{\mathrm{ii}}$ & $0.92(5)$ & $2.982(4)$ & $2.58(5)$ & $107(3)$ \\
$\mathrm{N}(1)-\mathrm{H}(1 \mathrm{~B}) \cdots \mathrm{O}(10)^{\mathrm{ii}}$ & $0.92(5)$ & $3.140(3)$ & $2.27(5)$ & $158(4)$ \\
$\mathrm{N}(2)-\mathrm{H}(2 \mathrm{~A}) \cdots \mathrm{O}(9)^{\mathrm{iii}}$ & $0.94(4)$ & $2.826(3)$ & $2.09(5)$ & $134(4)$ \\
$\mathrm{N}(2)-\mathrm{H}(2 \mathrm{~B}) \cdots \mathrm{O}(4)$ & $0.95(4)$ & $3.096(3)$ & $2.55(4)$ & $117(2)$ \\
$\mathrm{N}(2)-\mathrm{H}(2 \mathrm{~B}) \cdots \mathrm{O}(7)$ & $0.95(4)$ & $2.769(3)$ & $1.82(4)$ & $173(3)$ \\
\hline
\end{tabular}

Symmetry codes: (ii) $x-1 / 2,-y+2 / 3, z-1 / 2$ (iii) $-x+2,-y+1,-z+1$

Table S4. Hydrogen bond parameters for AlOC-14.

\begin{tabular}{lllll}
\hline $\mathrm{D}-\mathrm{H} \cdots \mathrm{A}$ & $\mathrm{d}(\mathrm{D}-\mathrm{H})$ & $\mathrm{d}(\mathrm{D} \cdots \mathrm{A})$ & $\mathrm{d}(\mathrm{H} \cdots \mathrm{A})$ & $<(\mathrm{DHA})$ \\
\hline $\mathrm{N}(4)-\mathrm{H}(4 \mathrm{AA}) \cdots \mathrm{O}(27)$ & 0.89 & $2.728(4)$ & 1.87 & 161 \\
$\mathrm{~N}(2)-\mathrm{H}(2 \mathrm{~A}) \cdots \mathrm{O}(15 \mathrm{~B})$ & 0.89 & $2.62(2)$ & 1.73 & 176 \\
$\mathrm{~N}(2)-\mathrm{H}(2 \mathrm{~A}) \cdots \mathrm{O}(15 \mathrm{~A})$ & 0.89 & $2.815(19)$ & 1.95 & 165 \\
$\mathrm{~N}(2)-\mathrm{H}(2 \mathrm{~B}) \ldots \mathrm{O}(11)^{\mathrm{i}}$ & 0.89 & $2.835(3)$ & 1.96 & 167 \\
$\mathrm{~N}(2)-\mathrm{H}(2 \mathrm{~B}) \ldots \mathrm{O}(13)$ & 0.89 & $3.011(3)$ & 2.50 & 117 \\
$\mathrm{~N}(4)-\mathrm{H}(4 \mathrm{AB}) \cdots \mathrm{O}(9)^{\mathrm{iii}}$ & 0.89 & $2.996(5)$ & 2.40 & 125 \\
$\mathrm{~N}(6)-\mathrm{H}(6 \mathrm{~A}) \cdots \mathrm{O}(18 \mathrm{~B})$ & 0.89 & $2.888(12)$ & 2.23 & 130 \\
$\mathrm{~N}(6)-\mathrm{H}(6 \mathrm{~A}) \cdots \mathrm{O}(19)^{\mathrm{ii}}$ & 0.89 & $2.962(4)$ & 2.12 & 158 \\
$\mathrm{~N}(6)-\mathrm{H}(6 \mathrm{~A}) \cdots \mathrm{O}(18 \mathrm{~A})$ & 0.89 & $2.969(11)$ & 2.36 & 126 \\
$\mathrm{~N}(6)-\mathrm{H}(6 \mathrm{~B}) \cdots \mathrm{O}(6)^{\mathrm{iiv}}$ & 0.89 & $2.706(4)$ & 1.82 & 175 \\
$\mathrm{O}(10)-\mathrm{H}(10) \cdots \mathrm{O}(8)$ & 0.82 & $2.494(4)$ & 1.82 & 138 \\
$\mathrm{O}(22)-\mathrm{H}(22) \cdots \mathrm{O}(20)$ & 0.82 & $2.499(3)$ & 1.74 & 154 \\
\hline
\end{tabular}

Symmetry codes: (i) $-\mathrm{x}+1,-\mathrm{y}+1,-\mathrm{z}+1$ (ii) $-\mathrm{x},-\mathrm{y}+2,-\mathrm{z}$ (iii) $\mathrm{x}, 1+\mathrm{y}, \mathrm{z}$ (iv) -x, 2-y, 1-z

Table S5 Selected bond lengths $(\AA)$ and angles $\left(^{\circ}\right)$ for AlOC-13-Ln series.

\begin{tabular}{|l|l|l|l|}
\hline \multicolumn{2}{|c|}{ AlOC-13-Dy } \\
\hline Dy1-O2 & $2.297(6)$ & Al1-O10 & $1.919(4)$ \\
Dy1-O3 & $2.341(5)$ & Al1-O6 & $1.907(4)$ \\
Dy1-O3 & Al1-O5 & $1.869(5)$ \\
Dy1-O4 & $2.341(4)$ & Al1-O12 & $1.835(5)$ \\
Dy1-O4 & Al2-O8 & $1.834(5)$ \\
Dy1-O1 & $2.268(4)$ & Al2-O9 & $1.717(6)$ \\
Dy1-O1 & Al2-O10 & $1.918(4)$ \\
Al1-O7 & $2.268(4)$ & Al2-O6 & $1.969(5)$ \\
Al1-O7 & $2.273(5)$ & Al2-O11 & $1.847(5)$ \\
\hline
\end{tabular}




\begin{tabular}{|c|c|c|c|}
\hline O2-Dy1-O3 ${ }^{i}$ & $72.56(14)$ & O7-Al1-O10 & $90.94(19)$ \\
\hline O2-Dy1-O3 & $72.56(14)$ & O7 $7^{\mathrm{ii}}$-Al1-O6 & $90.0(2)$ \\
\hline $\mathrm{O}^{\mathrm{i}}-\mathrm{Dy} 1-\mathrm{O} 3$ & $145.1(3)$ & O7-Al1-O6 & 84.79 (19) \\
\hline O4-Dy1-O2 & $141.71(13)$ & O6-Al1-O10 & $173.9(2)$ \\
\hline O4-Dy1-O2 & $141.72(13)$ & O5-Al1-O7 & $172.2(2)$ \\
\hline O4-Dy1-O3 & $141.08(19)$ & O5-Al1-O7i & $94.4(2)$ \\
\hline O4-Dy1-O3 & 72.09 (18) & O5-Al1-O10 & $90.9(2)$ \\
\hline O4-Dy1-O3 ${ }^{\mathrm{i}}$ & $141.08(19)$ & O5-Al1-O6 & $92.8(2)$ \\
\hline O4-Dy1-O3 ${ }^{\mathrm{i}}$ & $72.09(18)$ & $\mathrm{O} 12^{\mathrm{ii}}-\mathrm{Al} 1-\mathrm{O} 7^{\mathrm{ii}}$ & $172.2(2)$ \\
\hline O4-Dy1-O4 & $76.6(3)$ & $\mathrm{O} 12^{\mathrm{ii}-\mathrm{A} 11-\mathrm{O} 7}$ & $94.6(2)$ \\
\hline$O 4^{\mathrm{i}}-\mathrm{Dy} 1-\mathrm{O} 1^{\mathrm{i}}$ & $106.9(2)$ & $\mathrm{O} 12^{\mathrm{ii}}-\mathrm{Al} 1-\mathrm{O} 10^{\mathrm{ii}}$ & $92.2(2)$ \\
\hline O4-Dy1-O1 & $106.9(2)$ & $\mathrm{O} 12^{\mathrm{ii}}-\mathrm{A} 11-\mathrm{O} 6$ & $92.5(2)$ \\
\hline O4-Dy1-O $1^{\mathrm{i}}$ & $85.3(2)$ & $\mathrm{O} 12^{\mathrm{ii}}-\mathrm{Al} 1-\mathrm{O} 5$ & $92.9(2)$ \\
\hline O4-Dy1-O1 & $85.3(2)$ & O8-Al2-O10 & $139.4(2)$ \\
\hline $\mathrm{O} 1^{\mathrm{i}-\mathrm{D} y 1-\mathrm{O} 2}$ & $82.36(15)$ & O8-A12-O6 & $82.2(2)$ \\
\hline O1-Dy1-O2 & $82.37(15)$ & O8-Al2-O11 & $86.9(2)$ \\
\hline O1-Dy1-O3 ${ }^{i}$ & $93.1(2)$ & O9-A12-O8 & $108.3(2)$ \\
\hline O1-Dy1-O3 & $82.3(2)$ & O9-A12-O10 & $112.2(2)$ \\
\hline $\mathrm{O} 1^{\mathrm{i}-\mathrm{D} y 1-\mathrm{O} 3}$ & $93.1(2)$ & O9-A12-O6 & $104.1(2)$ \\
\hline$O 1^{i}-D y 1-O 3^{i}$ & $82.3(2)$ & O9-A12-O11 & $103.2(2)$ \\
\hline O1-Dy1-O $1^{\mathrm{i}}$ & $164.7(3)$ & O10-Al2-O6 & 88.27 (19) \\
\hline O7-Al1-O7 & $78.2(2)$ & O11-A12-O10 & $83.84(19)$ \\
\hline 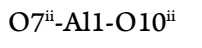 & $84.81(19)$ & O11-A12-O6 & $152.6(2)$ \\
\hline \multicolumn{4}{|c|}{ Symmetry codes: (i) $-x+1, y,-z+3 / 2$; (ii) $-x+3 / 2,-y+3 / 2,-z+1$; (iii) $-x+1, y,-z+1 / 2$} \\
\hline \multicolumn{4}{|c|}{ AlOC-13-Er } \\
\hline Er1-O10 & $2.246(3)$ & Al1-O3 & $1.921(3)$ \\
\hline Er1-O10 & $2.246(3)$ & Al1-O $7^{\mathrm{ii}}$ & $1.904(3)$ \\
\hline Er1-O13 & $2.283(4)$ & Al1-O2 & $1.833(3)$ \\
\hline $\operatorname{Er} 1-\mathrm{O} 12^{\mathrm{i}}$ & $2.328(3)$ & Al1-O9 ${ }^{\mathrm{ii}}$ & $1.859(3)$ \\
\hline Er1-O12 & $2.328(3)$ & $\mathrm{Al} 2-\mathrm{O} 3$ & $1.915(3)$ \\
\hline Er1-O11 $1^{\mathrm{i}}$ & $2.251(4)$ & $\mathrm{Al} 2-\mathrm{O} 4$ & $1.849(3)$ \\
\hline Er1-O11 & $2.251(4)$ & Al2-O7 & $1.968(3)$ \\
\hline $\mathrm{Al} 1-\mathrm{OS}^{\mathrm{ii}}$ & $1.865(3)$ & Al2-O6 & $1.734(4)$ \\
\hline Al1-O5 & $1.874(3)$ & $\mathrm{Al} 2-\mathrm{O} 8$ & $1.833(3)$ \\
\hline O10-Er1-O10 & $76.14(17)$ & $\mathrm{O}^{\mathrm{ii}}-\mathrm{All}-\mathrm{O} 3$ & $91.11(13)$ \\
\hline O10-Er1-O13 & $141.93(9)$ & $\mathrm{O} 5^{\mathrm{ii}}-\mathrm{Al} 1-\mathrm{O} 7^{\mathrm{ii}}$ & $84.75(13)$ \\
\hline O10-Er1-O13 & $141.93(8)$ & O5-Al1-O7ii & $89.77(13)$ \\
\hline $\mathrm{O} 10^{\mathrm{i}}-\mathrm{Er} 1-\mathrm{O} 12$ & $72.43(13)$ & $\mathrm{O} 7^{\mathrm{ii}-\mathrm{Al} 1-\mathrm{O} 3}$ & $173.70(14)$ \\
\hline $\mathrm{O} 10^{\mathrm{i}}-\mathrm{Er} 1-\mathrm{O} 12^{\mathrm{i}}$ & $140.53(14)$ & O2-Al1-O5 & $172.06(15)$ \\
\hline O10-Er1-O12 & $72.43(13)$ & $\mathrm{O} 2-\mathrm{Al1}-\mathrm{OS}^{\mathrm{ii}}$ & $94.92(14)$ \\
\hline O10-Er1-O12 & $140.53(14)$ & O2-Al1-O3 & $92.31(14)$ \\
\hline O10-Er1-O11 & $84.75(14)$ & O2-Al1-O $7^{\mathrm{ii}}$ & $92.77(14)$ \\
\hline O10-Er1-O11 & $107.38(14)$ & O2-Al1-O9 $9^{\mathrm{ii}}$ & $92.95(14)$ \\
\hline O10-Er1-O11 & $84.75(14)$ & O9ii-Al1-O5 & $94.45(14)$ \\
\hline
\end{tabular}




\begin{tabular}{|c|c|c|c|}
\hline O10-Er1-O11 $1^{\mathrm{i}}$ & $107.38(14)$ & $\mathrm{O} 9^{\mathrm{ii}}-\mathrm{Al} 1-\mathrm{O} 5^{\mathrm{ii}}$ & $171.82(15)$ \\
\hline O13-Er1-O12 & $72.63(10)$ & $\mathrm{O} 9^{\mathrm{ii}}-\mathrm{Al1}-\mathrm{O} 3$ & $90.78(13)$ \\
\hline $\mathrm{O} 13-\mathrm{Er} 1-\mathrm{O} 12^{\mathrm{i}}$ & $72.63(10)$ & $\mathrm{O} 9^{\mathrm{ii}}-\mathrm{Al} 1-\mathrm{O} 7^{\mathrm{ii}}$ & $92.67(14)$ \\
\hline O12-Er1-O12 & $145.3(2)$ & O3-A12-O7 & $88.24(13)$ \\
\hline O11-Er1-O13 & $82.44(10)$ & $\mathrm{O} 4-\mathrm{Al} 2-\mathrm{O} 3$ & $83.66(14)$ \\
\hline O11-Er1-O13 & $82.44(10)$ & O4-A12-O7 & $152.21(16)$ \\
\hline $\mathrm{O} 11^{\mathrm{i}}-\mathrm{Er} 1-\mathrm{O} 12^{\mathrm{i}}$ & $82.47(14)$ & O6-A12-O3 & $112.23(15)$ \\
\hline O11-Er1-O12 & $93.00(15)$ & O6-Al2-O4 & $103.37(16)$ \\
\hline 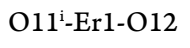 & $93.00(14)$ & O6-A12-O7 & $104.31(15)$ \\
\hline O11-Er1-O12 & $82.47(14)$ & O6-A12-O8 & $108.42(16)$ \\
\hline O11 - -Er1-O11 & $164.9(2)$ & $\mathrm{O} 8-\mathrm{Al} 2-\mathrm{O} 3$ & $139.35(16)$ \\
\hline $\mathrm{O}^{\mathrm{ii}-\mathrm{Al} 1-\mathrm{O} 5}$ & $77.81(14)$ & O8-A12-O4 & $86.89(14)$ \\
\hline O5-Al1-O3 & $84.71(13)$ & O8-Al2-O7 & $82.08(13)$ \\
\hline \multicolumn{4}{|c|}{ Symmetry codes: (i) $-x, y,-z+3 / 2$; (ii) $-x+1 / 2,-y+3 / 2,-z+1$; (iii) $-x, y,-z+1 / 2$. } \\
\hline \multicolumn{4}{|c|}{$\mathrm{AlOC}-13-\mathrm{Eu}$} \\
\hline Eu1-O4 & $2.298(3)$ & $\mathrm{A} 12-\mathrm{O} 10$ & $1.836(3)$ \\
\hline $\mathrm{Eu} 1-\mathrm{O} 4^{\mathrm{i}}$ & $2.298(3)$ & $\mathrm{Al2}-\mathrm{O} 12$ & $1.926(3)$ \\
\hline Eu1-O2 & $2.344(4)$ & Al2-O6 & $1.907(3)$ \\
\hline Eu1-O1 & $2.389(3)$ & Al2-O5 & $1.862(3)$ \\
\hline $\mathrm{Eu} 1-\mathrm{O} 1^{\mathrm{i}}$ & $2.389(3)$ & $\mathrm{Al1}-\mathrm{O} 12^{\mathrm{ii}}$ & $1.912(3)$ \\
\hline Eu1-O3 & $2.320(3)$ & Al1-O13 ${ }^{\mathrm{ii}}$ & $1.850(3)$ \\
\hline $\mathrm{Eu} 1-\mathrm{O} 3^{\mathrm{i}}$ & $2.320(3)$ & Al1-O6 & $1.962(3)$ \\
\hline Al2-O9 & $1.863(3)$ & Al1-O7 & $1.828(3)$ \\
\hline $\mathrm{Al} 2-\mathrm{O} 9^{\mathrm{ii}}$ & $1.869(3)$ & Al1-O8 & $1.731(3)$ \\
\hline O4-Eu1-O4 & $76.98(15)$ & O9-Al2-O12 & $91.16(12)$ \\
\hline O4-Eu1-O2 & $141.51(7)$ & $\mathrm{O} 99^{\mathrm{ii}}-\mathrm{Al} 2-\mathrm{O} 6$ & $89.85(12)$ \\
\hline $\mathrm{O} 4^{\mathrm{i}}-\mathrm{Eu} 1-\mathrm{O} 2$ & $141.51(7)$ & O9-Al2-O6 & $84.90(12)$ \\
\hline O4-Eu1-O1 & $72.35(11)$ & $\mathrm{O} 10-\mathrm{A} 12-\mathrm{O}^{\mathrm{ii}}$ & $172.21(13)$ \\
\hline O4-Eu1-O $1^{i}$ & $72.35(11)$ & O10-Al2-O9 & $94.89(13)$ \\
\hline $\mathrm{O} 4^{\mathrm{i}}-\mathrm{Eu} 1-\mathrm{O} 1^{\mathrm{i}}$ & $141.65(11)$ & $\mathrm{O} 10-\mathrm{A} 12-\mathrm{O} 12$ & $92.06(12)$ \\
\hline O4-Eu1-O1 & $141.65(11)$ & O10-Al2-O6 & $92.91(12)$ \\
\hline O4-Eu1-O3 & $107.26(12)$ & O10-Al2-O5 & $92.88(13)$ \\
\hline 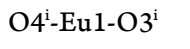 & $85.08(13)$ & O6-Al2-O12 & $173.90(12)$ \\
\hline O4-Eu1-O3 & $85.08(13)$ & O5-Al2-O9 & $94.27(12)$ \\
\hline O4-Eu1-O3 ${ }^{\mathrm{i}}$ & $107.26(12)$ & O5-A12-O9 & $171.97(13)$ \\
\hline $\mathrm{O} 2-\mathrm{Eu} 1-\mathrm{O} 1^{\mathrm{i}}$ & $72.11(9)$ & O5-Al2-O12 & $90.57(12)$ \\
\hline O2-Eu1-O1 & $72.11(9)$ & O5-A12-O6 & $92.71(12)$ \\
\hline O1-Eu1-O $1^{i}$ & $144.21(17)$ & $\mathrm{O} 12^{\mathrm{ii}}-\mathrm{A} 11-\mathrm{O} 6$ & $88.27(11)$ \\
\hline $\mathrm{O}^{\mathrm{i}}-\mathrm{Eu} 1-\mathrm{O} 2$ & $82.25(9)$ & 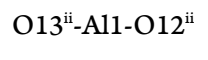 & $83.69(12)$ \\
\hline O3-Eu1-O2 & $82.25(9)$ & O13 ${ }^{\mathrm{ii}-\mathrm{A} 11-\mathrm{O} 6}$ & $152.83(14)$ \\
\hline O3-Eu1-O $1^{i}$ & $92.61(12)$ & O7-Al1-O12 $2^{\mathrm{ii}}$ & $139.60(14)$ \\
\hline O3-Eu1-O1 & $82.62(12)$ & O7-Al1-O13 $3^{\mathrm{ii}}$ & $86.92(13)$ \\
\hline 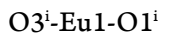 & $82.62(12)$ & O7-Al1-O6 & $82.52(12)$ \\
\hline O3-Eu1-O1 & $92.61(12)$ & $\mathrm{O} 8-\mathrm{Al} 1-\mathrm{O} 12^{\mathrm{ii}}$ & $112.57(13)$ \\
\hline
\end{tabular}




\begin{tabular}{|c|c|c|c|}
\hline O3-Eu1-O3 ${ }^{i}$ & $164.51(19)$ & O8-Al1-O13 ${ }^{i \mathrm{i}}$ & $102.97(15)$ \\
\hline O9-Al2-O9 $9^{\mathrm{ii}}$ & 78.09 (13) & O8-Al1-O6 & $104.09(14)$ \\
\hline $\mathrm{O} 9^{\mathrm{ii}}-\mathrm{A} 12-\mathrm{O} 12$ & $84.78(11)$ & O8-Al1-O7 & $107.83(14)$ \\
\hline \multicolumn{4}{|c|}{ Symmetry codes: (i) $-x+2, y,-z+1 / 2$; (ii) $-x+3 / 2,-y+3 / 2,-z+1$; (iii) $-x+1, y,-z+1 / 2$. } \\
\hline \multicolumn{4}{|c|}{ AlOC-13-Ho } \\
\hline Ho01-O4 & $2.252(4)$ & $\mathrm{Al2}-\mathrm{O} 10$ & $1.923(3)$ \\
\hline $\mathrm{Ho01-O} 4^{\mathrm{i}}$ & $2.252(4)$ & $\mathrm{Al2}-\mathrm{O} 11$ & $1.832(3)$ \\
\hline Ho01-O1 & $2.294(5)$ & Al2-O6 & $1.903(3)$ \\
\hline $\mathrm{Ho} 01-\mathrm{O} 2^{\mathrm{i}}$ & $2.337(4)$ & Al2-O5 & $1.868(4)$ \\
\hline Ho01-O2 & $2.337(4)$ & $\mathrm{Al} 1-\mathrm{O} 10^{\mathrm{ii}}$ & $1.920(3)$ \\
\hline $\mathrm{Ho} 1-\mathrm{O} 3^{\mathrm{i}}$ & $2.270(4)$ & Al1-O13 $3^{\mathrm{ii}}$ & $1.850(4)$ \\
\hline Ho01-O3 & $2.270(4)$ & Al1-O7 & $1.830(4)$ \\
\hline Al2-O9 & $1.869(4)$ & Al1-O6 & $1.967(3)$ \\
\hline $\mathrm{Al} 2-\mathrm{O} 9^{\mathrm{ii}}$ & $1.873(3)$ & Al1-O8 & $1.712(4)$ \\
\hline $\mathrm{O} 4-\mathrm{Ho} 01-\mathrm{O} 4^{\mathrm{i}}$ & $\mathrm{O} 4-\mathrm{Ho} 01-\mathrm{O} 4{ }^{\mathrm{i}}$ & O9-Al2-O10 & $90.96(15)$ \\
\hline O4-Ho01-O1 & O4-Ho01-O1 & $\mathrm{O} 9{ }^{\mathrm{ii}}-\mathrm{Al} 2-\mathrm{O} 6$ & $89.91(14)$ \\
\hline $\mathrm{O} 4 \mathrm{i}-\mathrm{Ho} 01-\mathrm{O} 1$ & O4-Ho01-O1 & O9-Al2-O6 & $84.70(14)$ \\
\hline $\mathrm{O} 4-\mathrm{Ho} 01-\mathrm{O} 2^{\mathrm{i}}$ & $\mathrm{O} 4-\mathrm{Ho} 01-\mathrm{O} 2^{\mathrm{i}}$ & O11-Al2-O9ii & $171.77(17)$ \\
\hline $\mathrm{O} 44^{\mathrm{i}}-\mathrm{Ho} 01-\mathrm{O} 2^{\mathrm{i}}$ & $\mathrm{O} 4^{\mathrm{i}}-\mathrm{Ho} 01-\mathrm{O} 2^{\mathrm{i}}$ & O11-Al2-O9 & $94.59(16)$ \\
\hline O4-Ho01-O2 & O4-Ho01-O2 & O11-A12-O10 & $92.01(15)$ \\
\hline $\mathrm{O} 4$ - $-\mathrm{Ho} 01-\mathrm{O} 2$ & $\mathrm{O} 4$-Ho01-O2 & O11-Al2-O6 & $92.90(14)$ \\
\hline $\mathrm{O} 4^{\mathrm{i}}-\mathrm{Ho} 01-\mathrm{O} 3^{\mathrm{i}}$ & $\mathrm{O} 4^{\mathrm{i}}-\mathrm{Ho} 01-\mathrm{O}^{\mathrm{i}}$ & O11-Al2-O5 & $93.35(16)$ \\
\hline $\mathrm{O} 4$ - $-\mathrm{Ho} 01-\mathrm{O} 3$ & O4-Ho01-O3 & O6-Al2-O10 & $173.69(15)$ \\
\hline O4-Ho01-O3 ${ }^{\mathrm{i}}$ & O4-Ho01-O3 ${ }^{\mathrm{i}}$ & $\mathrm{O} 5-\mathrm{Al} 2-\mathrm{O}^{\mathrm{ii}}$ & $94.23(15)$ \\
\hline O4-Ho01-O3 & O4-Ho01-O3 & O5-A12-O9 & $171.79(16)$ \\
\hline $\mathrm{O} 1-\mathrm{Ho} 01-\mathrm{O} 2^{\mathrm{i}}$ & $\mathrm{O} 1-\mathrm{Ho} 01-\mathrm{O} 2^{\mathrm{i}}$ & O5-Al2-O10 & $90.83(15)$ \\
\hline O1-Ho01-O2 & $\mathrm{O} 1-\mathrm{Ho} 01-\mathrm{O} 2$ & O5-Al2-O6 & $92.83(15)$ \\
\hline $\mathrm{O} 22^{\mathrm{i}}-\mathrm{Ho} 01-\mathrm{O} 2$ & $\mathrm{O} 2$ - $-\mathrm{Ho} 01-\mathrm{O} 2$ & O10 $10^{\mathrm{ii}}-\mathrm{A} 11-\mathrm{O} 6$ & $88.00(14)$ \\
\hline O3-Ho01-O1 & O3-Ho01-O1 & $\mathrm{O} 13^{\mathrm{ii}}-\mathrm{A} 11-\mathrm{O} 10^{\mathrm{ii}}$ & $83.67(14)$ \\
\hline O3 ${ }^{\mathrm{i}}-\mathrm{Ho} 01-\mathrm{O} 1$ & O3 $-\mathrm{Ho} 01-\mathrm{O} 1$ & O13 ${ }^{\mathrm{ii}}$-Al1-O6 & $152.41(18)$ \\
\hline $\mathrm{O} 33^{\mathrm{i}}-\mathrm{Ho} 01-\mathrm{O} 2$ & 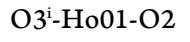 & O7-Al1-O10 & $139.24(18)$ \\
\hline $\mathrm{O} 3^{\mathrm{i}}-\mathrm{Ho} 01-\mathrm{O} 2^{\mathrm{i}}$ & $\mathrm{O} 3^{\mathrm{i}}-\mathrm{Ho} 01-\mathrm{O} 2^{\mathrm{i}}$ & $\mathrm{O} 7-\mathrm{Al} 1-\mathrm{O} 13^{\mathrm{ii}}$ & $87.11(15)$ \\
\hline O3-Ho01-O2 & O3-Ho01-O2 & O7-Al1-O6 & $82.17(15)$ \\
\hline $\mathrm{O} 3-\mathrm{Ho} 01-\mathrm{O} 2^{\mathrm{i}}$ & $\mathrm{O} 3-\mathrm{Ho} 01-\mathrm{O} 2^{\mathrm{i}}$ & O8-Al1-O10 ${ }^{\mathrm{ii}}$ & $112.32(16)$ \\
\hline O3-Ho01-O3 & O3-Ho01-O3 & O8-Al1-O13 ${ }^{\mathrm{ii}}$ & $103.15(18)$ \\
\hline O9-Al2-O9ii & O9-Al2-O9ii & O8-Al1-O7 & $108.44(18)$ \\
\hline $\mathrm{O} 9{ }^{\mathrm{ii}}-\mathrm{A} 12-\mathrm{O} 10$ & $\mathrm{O} 9{ }^{\mathrm{ii}}-\mathrm{A} 12-\mathrm{O} 10$ & O8-Al1-O6 & $104.34(16)$ \\
\hline \multicolumn{4}{|c|}{ Symmetry codes: (i) $-x+2, y,-z+1 / 2$; (ii) $-x+3 / 2,-y+3 / 2,-z+1$; (iii) $-x+1, y,-z+1 / 2$. } \\
\hline \multicolumn{4}{|c|}{$\mathrm{AlOC}-13-\mathrm{Sm}$} \\
\hline Sm1-O1 & $2.360(5)$ & Al2-O7 & $1.928(4)$ \\
\hline Sm1-O4 & $2.316(4)$ & Al2-O6 & $1.839(4)$ \\
\hline $\mathrm{Sm} 1-\mathrm{O} 4^{\mathrm{i}}$ & $2.316(4)$ & $\mathrm{Al2}-\mathrm{O} 12$ & $1.906(4)$ \\
\hline $\mathrm{Sm} 1-\mathrm{O} 2$ & $2.412(4)$ & $\mathrm{Al2}-\mathrm{O} 13$ & $1.855(4)$ \\
\hline $\mathrm{Sm} 1-\mathrm{O} 2^{\mathrm{i}}$ & $2.412(4)$ & Al1-O7 ${ }^{i \mathrm{i}}$ & $1.916(4)$ \\
\hline
\end{tabular}




\begin{tabular}{|c|c|c|c|}
\hline $\mathrm{Sm} 1-\mathrm{O}^{\mathrm{i}}$ & $2.310(5)$ & Al1-O ${ }^{i i}$ & $1.848(4)$ \\
\hline Sm1-O3 & $2.310(5)$ & Al1-O11 & $1.829(4)$ \\
\hline Al2-O9 & $1.864(4)$ & Al1-O12 & $1.968(4)$ \\
\hline $\mathrm{Al} 2-\mathrm{O}^{\mathrm{ii}}$ & $1.870(4)$ & Al1-O10 & $1.739(4)$ \\
\hline $\mathrm{O} 1-\mathrm{Sm} 1-\mathrm{O} 2^{\mathrm{i}}$ & $71.78(11)$ & $\mathrm{O} 99^{\mathrm{ii}}-\mathrm{A} 12-\mathrm{O} 7$ & $84.70(15)$ \\
\hline $\mathrm{O} 1-\mathrm{Sm} 1-\mathrm{O} 2$ & $71.78(11)$ & $\mathrm{O} 9^{\mathrm{ii}}-\mathrm{A} 12-\mathrm{O} 12$ & $89.87(16)$ \\
\hline $\mathrm{O} 4 \mathrm{i}-\mathrm{Sm} 1-\mathrm{O} 1$ & $141.49(10)$ & O9-Al2-O12 & $84.72(16)$ \\
\hline O4-Sm1-O1 & $141.50(10)$ & O6-A12-O9 & $94.80(17)$ \\
\hline $\mathrm{O} 4-\mathrm{Sm} 1-\mathrm{O} 4^{\mathrm{i}}$ & $77.01(19)$ & $\mathrm{O} 6-\mathrm{A} 12-\mathrm{O}^{\mathrm{ii}}$ & $172.01(17)$ \\
\hline $\mathrm{O} 4-\mathrm{Sm} 1-\mathrm{O} 2$ & $142.39(15)$ & O6-A12-O7 & $92.16(17)$ \\
\hline $\mathrm{O} 4^{\mathrm{i}}-\mathrm{Sm} 1-\mathrm{O} 2^{\mathrm{i}}$ & $142.39(15)$ & O6-Al2-O12 & $92.86(16)$ \\
\hline $\mathrm{O} 4 \mathrm{i}-\mathrm{Sm} 1-\mathrm{O} 2$ & 72.37 (14) & O6-Al2-O13 & $93.11(17)$ \\
\hline $\mathrm{O} 4-\mathrm{Sm} 1-\mathrm{O} 2^{\mathrm{i}}$ & $72.38(14)$ & O12-Al2-O7 & $173.88(17)$ \\
\hline $\mathrm{O} 2{ }^{\mathrm{i}}-\mathrm{Sm} 1-\mathrm{O} 2$ & $143.6(2)$ & $\mathrm{O} 13-\mathrm{Al} 2-\mathrm{O} 9^{\mathrm{ii}}$ & $94.28(16)$ \\
\hline O3-Sm1-O1 & $82.23(12)$ & O13-Al2-O9 & $171.71(18)$ \\
\hline $\mathrm{O}^{\mathrm{i}}-\mathrm{Sm} 1-\mathrm{O} 1$ & $82.23(12)$ & O13-Al2-O7 & $90.82(16)$ \\
\hline $\mathrm{O}^{\mathrm{i}}-\mathrm{Sm} 1-\mathrm{O} 4$ & $106.92(16)$ & O13-A12-O12 & $92.40(16)$ \\
\hline O3-Sm1-O4i & $106.92(16)$ & $\mathrm{O} 7^{\mathrm{ii}-\mathrm{A} 11-\mathrm{O} 12}$ & $88.15(15)$ \\
\hline $\mathrm{O} 3^{\mathrm{i}}-\mathrm{Sm} 1-\mathrm{O} 4^{\mathrm{i}}$ & $85.44(16)$ & $\mathrm{O}^{\mathrm{ii}}-\mathrm{Al} 1-\mathrm{O} 7^{\mathrm{ii}}$ & $83.65(16)$ \\
\hline O3-Sm1-O4 & $85.44(16)$ & $\mathrm{O} 8^{\mathrm{i}-\mathrm{A}} \mathrm{Al}-\mathrm{O} 12$ & $152.95(19)$ \\
\hline $\mathrm{O} 3-\mathrm{Sm} 1-\mathrm{O} 2^{\mathrm{i}}$ & $92.02(16)$ & O11-Al1-O7 ${ }^{i \mathrm{i}}$ & $139.29(18)$ \\
\hline $\mathrm{O} 3-\mathrm{Sm} 1-\mathrm{O} 2$ & $83.12(16)$ & O11-Al1-O $8^{\mathrm{ii}}$ & $87.05(17)$ \\
\hline $\mathrm{O}^{\mathrm{i}}-\mathrm{Sm} 1-\mathrm{O} 2$ & $92.02(16)$ & O11-Al1-O12 & 82.49 (16) \\
\hline $\mathrm{O} 3^{\mathrm{i}}-\mathrm{Sm} 1-\mathrm{O} 2^{\mathrm{i}}$ & $83.12(16)$ & O10-Al1-O7 ${ }^{\mathrm{ii}}$ & $112.66(17)$ \\
\hline $\mathrm{O}^{\mathrm{i}}-\mathrm{Sm} 1-\mathrm{O} 3$ & $164.5(3)$ & O10-Al1-O $8^{\mathrm{ii}}$ & $103.04(19)$ \\
\hline O9-Al2-O9ii & $77.97(17)$ & O10-Al1-O11 & $108.06(19)$ \\
\hline O9-Al2-O7 & $91.37(16)$ & O10-Al1-O12 & $103.91(18)$ \\
\hline \multicolumn{4}{|c|}{ Symmetry codes: (i) $-x+2, y,-z+1 / 2$; (ii) $-x+3 / 2,-y+3 / 2,-z+1$; (iii) $-x+1, y,-z+1 / 2$. } \\
\hline \multicolumn{4}{|c|}{ AlOC-13-Tb } \\
\hline $\mathrm{Tb} 1-\mathrm{O} 4^{\mathrm{i}}$ & $2.282(3)$ & All-O11 ${ }^{\text {ii }}$ & $1.865(3)$ \\
\hline Tb1-O4 & $2.282(3)$ & Al1-O9 & $1.922(3)$ \\
\hline Tb1-O2 & $2.321(4)$ & Al1-O5 $5^{\mathrm{ii}}$ & $1.861(3)$ \\
\hline $\mathrm{Tb} 1-\mathrm{O} 1^{\mathrm{i}}$ & $2.365(3)$ & Al1-O6 $6^{\mathrm{ii}}$ & $1.908(3)$ \\
\hline Tb1-O1 & $2.365(3)$ & Al2-O9 & $1.915(3)$ \\
\hline $\mathrm{Tb} 1-\mathrm{O}^{\mathrm{i}}$ & $2.288(3)$ & $\mathrm{Al} 2-\mathrm{O} 10$ & $1.737(3)$ \\
\hline Tb1-O3 & $2.288(3)$ & $\mathrm{Al} 2-\mathrm{O} 8$ & $1.849(3)$ \\
\hline Al1-O12 & $1.836(3)$ & Al2-O6 & $1.965(3)$ \\
\hline Al1-O11 & $1.871(3)$ & $\mathrm{Al} 2-\mathrm{O} 7$ & $1.826(3)$ \\
\hline $\mathrm{O} 4 \mathrm{i}-\mathrm{Tb} 1-\mathrm{O} 4$ & $76.45(15)$ & O12-Al1-O9 & $92.10(12)$ \\
\hline $\mathrm{O} 4-\mathrm{Tb} 1-\mathrm{O} 2$ & $141.77(7)$ & O12-Al1-O5 ${ }^{\mathrm{ii}}$ & $92.86(13)$ \\
\hline $\mathrm{O} 4^{\mathrm{i}-\mathrm{Tb} 1-\mathrm{O} 2}$ & $141.78(7)$ & O12-Al1-O6 ${ }^{\mathrm{ii}}$ & $92.97(12)$ \\
\hline O4-Tb1-O1 & $141.38(12)$ & 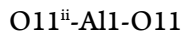 & $77.97(13)$ \\
\hline $\mathrm{O} 4-\mathrm{Tb} 1-\mathrm{O} 1^{\mathrm{i}}$ & $72.41(11)$ & O11 $11^{\mathrm{ii}} \mathrm{Al1}-\mathrm{O} 9$ & $91.18(12)$ \\
\hline $\mathrm{O} 4 \mathrm{i}-\mathrm{Tb} 1-\mathrm{O} 1$ & $72.41(11)$ & O11-Al1-O9 & $84.69(12)$ \\
\hline
\end{tabular}




\begin{tabular}{|c|c|c|c|}
\hline $\mathrm{O} 4^{\mathrm{i}}-\mathrm{Tb} 1-\mathrm{O} 1^{\mathrm{i}}$ & $141.38(12)$ & $\mathrm{O} 11-\mathrm{A} 11-\mathrm{O} 6^{\mathrm{ii}}$ & $89.81(12)$ \\
\hline $\mathrm{O} 4-\mathrm{Tb} 1-\mathrm{O} 3^{\mathrm{i}}$ & $107.03(12)$ & $\mathrm{O} 11^{\mathrm{ii}-\mathrm{A} l 1-\mathrm{O} 6^{\mathrm{ii}}}$ & $84.75(12)$ \\
\hline $\mathrm{O} 4-\mathrm{Tb} 1-\mathrm{O} 3$ & $85.17(12)$ & $\mathrm{O} 5^{\mathrm{ii}}-\mathrm{Al} 1-\mathrm{O} 11^{\mathrm{ii}}$ & $171.94(14)$ \\
\hline 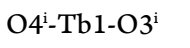 & $85.17(12)$ & $\mathrm{O}^{\mathrm{ii}-\mathrm{A}} 11-\mathrm{O} 11$ & $94.40(13)$ \\
\hline 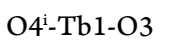 & $107.03(12)$ & $\mathrm{O}^{\mathrm{ii}-\mathrm{A} 11-\mathrm{O} 9}$ & $90.66(12)$ \\
\hline $\mathrm{O} 2-\mathrm{Tb} 1-\mathrm{O} 1^{\mathrm{i}}$ & $72.25(8)$ & $\mathrm{O} 5^{\mathrm{ii}}-\mathrm{Al} 1-\mathrm{O} 6^{\mathrm{ii}}$ & $92.72(12)$ \\
\hline $\mathrm{O} 2-\mathrm{Tb} 1-\mathrm{O} 1$ & $72.25(8)$ & O66-All-O9 & $173.75(13)$ \\
\hline 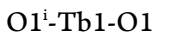 & $144.50(17)$ & O9-Al2-O6 & $88.15(12)$ \\
\hline O3-Tb1-O2 & $82.37(9)$ & O10-Al2-O9 & $112.19(13)$ \\
\hline $\mathrm{O}^{\mathrm{i}}-\mathrm{Tb} 1-\mathrm{O} 2$ & $82.37(9)$ & O10-Al2-O8 & $102.96(14)$ \\
\hline $\mathrm{O} 3-\mathrm{Tb} 1-\mathrm{O} 1^{\mathrm{i}}$ & $92.65(12)$ & O10-Al2-O6 & $104.29(14)$ \\
\hline $\mathrm{O} 3^{\mathrm{i}}-\mathrm{Tb} 1-\mathrm{O} 1^{\mathrm{i}}$ & $82.69(12)$ & O10-Al2-O7 & $108.30(14)$ \\
\hline $\mathrm{O}^{\mathrm{i}}-\mathrm{Tb} 1-\mathrm{O} 1$ & $92.65(12)$ & O8-Al2-O9 & $83.78(12)$ \\
\hline O3-Tb1-O1 & $82.69(12)$ & O8-Al2-O6 & $152.65(14)$ \\
\hline O3-Tb1-O3 & $164.74(18)$ & O7-Al2-O9 & $139.51(14)$ \\
\hline O12-Al1-O11 & $172.09(13)$ & O7-Al2-O8 & $87.15(13)$ \\
\hline O12-Al1-O11 ${ }^{\text {ii }}$ & $94.90(13)$ & O7-Al2-O6 & $82.16(12)$ \\
\hline \multicolumn{4}{|c|}{ Symmetry codes: (i) $-x, y,-z+3 / 2$; (ii) $-x+1 / 2,-y+3 / 2,-z+1$; (iii) $-x, y,-z+1 / 2$. } \\
\hline \multicolumn{4}{|c|}{ AlOC-13-Gd } \\
\hline Gd1-O4 & $2.288(3)$ & Al1-O9ii & $1.866(3)$ \\
\hline $\mathrm{Gd} 1-\mathrm{O} 4^{\mathrm{i}}$ & $2.288(3)$ & Al1-O10 & $1.926(3)$ \\
\hline Gd1-O1 & $2.328(5)$ & Al1-O6 ${ }^{\mathrm{ii}}$ & $1.907(3)$ \\
\hline $\mathrm{Gd} 1-\mathrm{O} 2$ & $2.373(3)$ & $\mathrm{Al} 1-\mathrm{O} 5^{\mathrm{ii}}$ & $1.859(3)$ \\
\hline $\mathrm{Gd} 1-\mathrm{O} 2^{\mathrm{i}}$ & $2.373(3)$ & $\mathrm{Al2}-\mathrm{O} 10$ & $1.914(3)$ \\
\hline $\mathrm{Gd}^{1-O} 3^{\mathrm{i}}$ & $2.303(4)$ & $\mathrm{Al} 2-\mathrm{O} 11$ & $1.846(4)$ \\
\hline Gd1-O3 & $2.303(4)$ & $\mathrm{Al} 2-\mathrm{O} 7$ & $1.828(3)$ \\
\hline Al1-O12 & $1.837(3)$ & $\mathrm{Al} 2-\mathrm{O} 6$ & $1.966(3)$ \\
\hline Al1-O9 & $1.874(3)$ & $\mathrm{Al} 2-\mathrm{O} 8$ & $1.743(4)$ \\
\hline O4-Gd1-O4 & $76.60(18)$ & O12-Al1-O10 & $92.13(14)$ \\
\hline O4-Gd1-O1 & $141.70(9)$ & O12-Al1-O6 $6^{\mathrm{ii}}$ & $92.89(14)$ \\
\hline $\mathrm{O} 4 \mathrm{i}-\mathrm{Gd} 1-\mathrm{O} 1$ & $141.70(9)$ & O12-Al1-OS ${ }^{\mathrm{ii}}$ & $92.88(15)$ \\
\hline $\mathrm{O} 4 \mathrm{i}-\mathrm{Gd} 1-\mathrm{O} 2$ & $72.37(13)$ & O9i-Al1-O9 & $78.19(15)$ \\
\hline $\mathrm{O} 4-\mathrm{Gd} 1-\mathrm{O} 2^{\mathrm{i}}$ & $72.37(13)$ & O9 ${ }^{\mathrm{ii}}-\mathrm{A} 11-\mathrm{O} 10$ & $91.20(14)$ \\
\hline $\mathrm{O} 4^{\mathrm{i}}-\mathrm{Gd} 1-\mathrm{O} 2^{\mathrm{i}}$ & $141.25(14)$ & O9-Al1-O10 & $84.79(13)$ \\
\hline O4-Gd1-O2 & $141.25(14)$ & O9-Al1-O6 ${ }^{\mathrm{ii}}$ & $89.79(14)$ \\
\hline 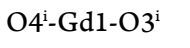 & $85.16(15)$ & $\mathrm{O} 9^{\mathrm{ii}}-\mathrm{Al} 1-\mathrm{O}^{\mathrm{ii}}$ & $84.89(13)$ \\
\hline $\mathrm{O} 4 \mathrm{i}-\mathrm{Gd} 1-\mathrm{O} 3$ & $107.33(14)$ & $\mathrm{O} 6^{\mathrm{ii}}-\mathrm{A} 11-\mathrm{O} 10$ & $173.88(14)$ \\
\hline O4-Gd1-O3 ${ }^{\mathrm{i}}$ & $107.33(14)$ & $\mathrm{O}^{\mathrm{ii}-\mathrm{Al} 1-\mathrm{O} 9}$ & $94.21(14)$ \\
\hline O4-Gd1-O3 & $85.16(15)$ & $\mathrm{O} 5^{\mathrm{ii}}-\mathrm{Al} 1-\mathrm{O} 9^{\mathrm{ii}}$ & $171.98(15)$ \\
\hline $\mathrm{O} 1-\mathrm{Gd} 1-\mathrm{O} 2^{\mathrm{i}}$ & $72.31(10)$ & $\mathrm{OS}^{\mathrm{ii}-\mathrm{A}} 11-\mathrm{O} 10$ & $90.62(14)$ \\
\hline O1-Gd1-O2 & $72.31(10)$ & $\mathrm{O}^{\mathrm{ii}-\mathrm{A} 11-\mathrm{O}^{\mathrm{ii}}}$ & $92.62(14)$ \\
\hline $\mathrm{O} 2-\mathrm{Gd} 1-\mathrm{O} 2^{\mathrm{i}}$ & $144.6(2)$ & O10-Al2-O6 & $88.28(13)$ \\
\hline O3-Gd1-O1 & $82.18(11)$ & $\mathrm{O} 11-\mathrm{Al} 2-\mathrm{O} 10$ & $83.60(13)$ \\
\hline 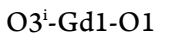 & $82.18(11)$ & O11-Al2-O6 & $152.89(16)$ \\
\hline
\end{tabular}




\begin{tabular}{|c|c|c|c|}
\hline 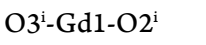 & $82.54(14)$ & O7-Al2-O10 & $139.58(17)$ \\
\hline $\mathrm{O} 3-\mathrm{Gd} 1-\mathrm{O} 2^{\mathrm{i}}$ & $92.71(15)$ & O7-Al2-O11 & $87.15(15)$ \\
\hline 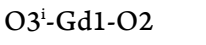 & $92.71(15)$ & O7-Al2-O6 & $82.40(14)$ \\
\hline O3-Gd1-O2 & $82.54(14)$ & O8-Al2-O10 & $112.26(15)$ \\
\hline O3-Gd1-O3 & $164.4(2)$ & O8-Al2-011 & $102.91(17)$ \\
\hline O12-Al1-O9 & $172.30(16)$ & O8-Al2-O7 & $108.16(16)$ \\
\hline O12-Al1-O9ii & $94.85(15)$ & O8-A12-06 & $104.11(16)$ \\
\hline \multicolumn{4}{|c|}{ Symmetry codes: (i) $-x, y,-z+3 / 2$; (ii) $-x+1 / 2,-y+3 / 2,-z+1$; (iii) $-x, y,-z+1 / 2$. } \\
\hline \multicolumn{4}{|c|}{ AlOC-13-Yb } \\
\hline $\mathrm{Yb} 1-\mathrm{O} 10^{\mathrm{i}}$ & $2.223(3)$ & $\mathrm{Al2}-\mathrm{O} 8$ & $1.832(3)$ \\
\hline Yb1-O10 & $2.223(3)$ & $\mathrm{Al} 2-\mathrm{O} 7$ & $1.980(3)$ \\
\hline Yb1-O13 & $2.260(4)$ & $\mathrm{Al2}-\mathrm{O} 6$ & $1.699(4)$ \\
\hline $\mathrm{Yb} 1-\mathrm{O} 12$ & $2.295(3)$ & Al1-O5 & $1.869(3)$ \\
\hline $\mathrm{Yb} 1-\mathrm{O} 12^{\mathrm{i}}$ & $2.295(3)$ & $\mathrm{All}_{-}-5^{\mathrm{ii}}$ & $1.872(3)$ \\
\hline Yb1-O11 ${ }^{\mathrm{i}}$ & $2.237(4)$ & Al1-O3 & $1.918(3)$ \\
\hline Yb1-O11 & 2.237 (4) & Al1-O2 & $1.838(3)$ \\
\hline $\mathrm{Al} 2-\mathrm{O} 3$ & $1.934(3)$ & All-O $7^{\mathrm{ii}}$ & $1.901(3)$ \\
\hline Al2-O4 & 1.849 (3) & Al1-O99 & $1.864(3)$ \\
\hline O10-Yb1-O10 & $75.68(17)$ & O4-Al2-O7 & $151.22(16)$ \\
\hline O10-Yb1-O13 & $142.16(8)$ & O8-Al2-O3 & $138.69(16)$ \\
\hline O10-Yb1-O13 & $142.16(8)$ & O8-Al2-O4 & $86.86(14)$ \\
\hline O10-Yb1-O12 & $140.41(13)$ & O8-Al2-O7 & 82.09 (13) \\
\hline O10-Yb1-O12i & $72.68(12)$ & O6-Al2-O3 & $112.35(14)$ \\
\hline O10 & $140.42(13)$ & O6-Al2-O4 & $103.78(16)$ \\
\hline O10-Yb1-O12 & $72.68(12)$ & O6-Al2-O8 & $108.96(16)$ \\
\hline O10-Yb1-O11 & $107.23(13)$ & O6-Al2-O7 & $104.93(15)$ \\
\hline O10-Yb1-O11 & $107.23(13)$ & O5-Al1-O5 ${ }^{\mathrm{ii}}$ & $77.90(14)$ \\
\hline O10 $-\mathrm{Yb} 1-\mathrm{O} 11^{\mathrm{i}}$ & $84.62(14)$ & $\mathrm{O}^{\mathrm{ii}}-\mathrm{All}-\mathrm{O} 3$ & $90.96(13)$ \\
\hline O10-Yb1-O11 & $84.62(14)$ & O5-Al1-O3 & 84.80 (13) \\
\hline O13-Yb1-O12 & $72.58(10)$ & O5-Al1-O $7^{\mathrm{ii}}$ & 89.89 (13) \\
\hline O13-Yb1-O12i & $72.58(10)$ & $\mathrm{O}^{\mathrm{ii}}-\mathrm{Al} 1-\mathrm{O} 7^{\mathrm{ii}}$ & $84.97(13)$ \\
\hline $\mathrm{O} 12^{\mathrm{i}-\mathrm{Yb} 1-\mathrm{O} 12}$ & $145.2(2)$ & O2-Al1-OS ${ }^{\mathrm{ii}}$ & $94.63(14)$ \\
\hline 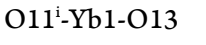 & $82.64(10)$ & O2-Al1-O5 & $171.85(15)$ \\
\hline O11-Yb1-O13 & $82.64(10)$ & O2-Al1-O3 & $92.14(13)$ \\
\hline $\mathrm{O} 11^{\mathrm{i}-\mathrm{Yb} 1-\mathrm{O} 12}$ & $92.92(14)$ & O2-Al1-O $7^{\mathrm{ii}}$ & $92.72(13)$ \\
\hline 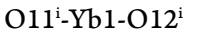 & $82.66(14)$ & O2-Al1-O9ii & 93.00 (14) \\
\hline O11-Yb1-O12 & $92.92(14)$ & O $7^{7 i}-A l 1-O 3$ & $173.90(13)$ \\
\hline O11-Yb1-O12 & $82.66(14)$ & O9ii-All-O5 & $94.59(14)$ \\
\hline O11-Yb1-O11 & $165.3(2)$ & $O 9^{\mathrm{ii}}-\mathrm{Al} 1-\mathrm{O} 5^{\mathrm{ii}}$ & $172.09(15)$ \\
\hline O3-Al2-O7 & $87.62(13)$ & $\mathrm{O} 9^{\mathrm{ii}}-\mathrm{All1}-\mathrm{O} 3$ & $90.81(13)$ \\
\hline O4-Al2-O3 & $83.31(13)$ & $\mathrm{O} 9^{\mathrm{ii}}-\mathrm{Al} 1-\mathrm{O} 7^{\mathrm{ii}}$ & $92.62(13)$ \\
\hline
\end{tabular}


Table S6. Hydrogen bond parameters for AlOC-13-Dy.

(due to the similarity of series AlOC-13-Ln, only AlOC-13-Dy is shown here)

\begin{tabular}{lllll}
\hline $\mathrm{D}-\mathrm{H} \cdots \mathrm{A}$ & $\mathrm{d}(\mathrm{D}-\mathrm{H})$ & $\mathrm{d}(\mathrm{D} \cdots \mathrm{A})$ & $\mathrm{d}(\mathrm{H} \cdots \mathrm{A})$ & $<(\mathrm{DHA})$ \\
\hline $\mathrm{O}(2)-\mathrm{H}(2 \mathrm{~A}) \cdots \mathrm{O}(8)^{\mathrm{ii}}$ & 0.85 & $2.609(6)$ & 1.81 & 155 \\
$\mathrm{O}(2)-\mathrm{H}(2 \mathrm{~B}) \cdots \mathrm{O}(8)^{\mathrm{i}}$ & 0.85 & $2.609(6)$ & 2.28 & 103 \\
$\mathrm{~N}(2)-\mathrm{H}(2 \mathrm{~F}) \cdots \mathrm{O}(13)$ & 0.89 & $2.747(8)$ & 1.86 & 172 \\
$\mathrm{~N}(2)-\mathrm{H}(2 \mathrm{G}) \cdots \mathrm{O}(13)^{\mathrm{iii}}$ & 0.89 & $2.747(8)$ & 1.86 & 172 \\
$\mathrm{O}(3)-\mathrm{H}(3 \mathrm{~A}) \cdots \mathrm{O}(5)$ & 0.85 & $3.255(7)$ & 2.57 & 138 \\
$\mathrm{O}(3)-\mathrm{H}(3 \mathrm{~B}) \cdots \mathrm{O}(2)$ & 0.85 & $2.744(6)$ & 2.39 & 106 \\
$\mathrm{O}(3)-\mathrm{H}(3 \mathrm{~B}) \cdots \mathrm{O}(11)^{\mathrm{i}}$ & 0.85 & $2.671(7)$ & 2.27 & 109 \\
\hline
\end{tabular}

Symmetry codes: (i) $\mathrm{x}-0.5,-\mathrm{y}+0.5$, z (ii) 1.5-x, $0.5+\mathrm{y}, 1.5-\mathrm{z}$ (iii) 1-x, y, 0.5-z 